DR. PAULINE ROUAUD-TINGUELY (Orcid ID : 0000-0003-2238-7077)

DR. JOSSELIN BREUGNOT (Orcid ID : 0000-0002-3459-8819)

Article type : Original Article

\title{
Shift in skin microbiota of Western European women across aging
}

Running title: Western European skin microbiota and aging

Romain Jugé ${ }^{1}$, Pauline Rouaud-Tinguely ${ }^{1 *}$, Josselin Breugnot ${ }^{1}$, Katia Servaes $^{1}$, Christine Grimaldi ${ }^{1}$, Marie-Paule Roth ${ }^{2}$, Hélène Coppin ${ }^{2}$ and Brigitte Closs $^{1}$

${ }^{1}$ R\&D department, SILAB, Brive-la-Gaillarde, France

${ }^{2}$ IRSD, Université de Toulouse, INSERM, INRA, ENUT, Toulouse, France

* Corresponding author: Dr Pauline Rouaud-Tinguely; mail: scientificom@silab.fr

Abbreviations: OTUs, operational taxonomic units; LEfSe, linear discriminant analysis effect size; LDA, linear discriminant analysis; PCoA, principal coordinates analysis

Funding statement: None

This article has been accepted for publication and undergone full peer review but has not been through the copyediting, typesetting, pagination and proofreading process, which may lead to differences between this version and the Version of Record. Please cite this article as doi: 10.1111/jam.13929

This article is protected by copyright. All rights reserved. 


\begin{abstract}
Aims The objective of our study was to compare the microbiota diversity between two different age groups of Western European women.

Methods and Results Skin-swab samples were collected directly on the forehead of 34 healthy Western European women: 17 younger (21-31 years old) and 17 older individuals (54-69 years old). Bacterial communities were evaluated using the 16S rRNA gene sequencing. Data revealed a higher alpha-diversity on the skin of older individuals compared to younger ones. Overall microbiota structure was different between the two age groups, as demonstrated by beta-diversity analysis, which also highlighted a high interpersonal variation within older individuals. Furthermore, taxonomic composition analysis showed both an increase of Proteobacteria and a decrease of Actinobacteria on the older skin. At the genus level, older skin exhibited a significant increase in Corynebacterium and a decrease in Propionibacterium relative abundance.
\end{abstract}

Conclusions Our study revealed a shift in the distribution of skin microbiota during chronological aging in Western European women.

Significance and Impact of Study Altogether these results could become the basis to develop new approaches aiming to rebalance the skin microbiota, which is modified during the aging process.

Keywords: skin, age-related changes, microbiota, 16S rRNA gene sequencing, alpha diversity, beta diversity, Western European women

This article is protected by copyright. All rights reserved. 


\section{Introduction}

The skin is the largest organ in the human body. It is composed of three organized layers i.e. epidermis, dermis and hypodermis - acting as a complex and essential barrier to protect the body against external aggressions, including pathogen invasion (Madison 2003). The skin also hosts a real ecosystem composed of various microorganisms including fungi, bacteria, viruses and archaea, reaching concentrations of 10 million of cells per square centimeter (Fredricks 2001; Grice and Segre 2011).

The majority of these microorganisms is harmless and even beneficial. They constitute the skin microbiota that is responsible for inhibiting pathogens adhesion and educating the host's local immune system (Sanford and Gallo 2013; Byrd et al. 2018). The intimate commensal relationship between the skin and its microbiota is very complex but necessary for healthy skin with efficient barrier function (Dréno et al. 2016; Baldwin et al. 2017). Indeed, several studies have demonstrated that an imbalance of skin microbiota is associated with various skin diseases such as psoriasis (Tett et al. 2017), vitiligo (Ganju et al. 2016), atopic dermatitis (Kong et al. 2012) or acne (Rocha and Bagatin 2017).

Among the diversity of skin microbes, the bacterial microbiota represents the most studied community. Four main phyla have been characterized in healthy skin: Actinobacteria, Firmicutes, Proteobacteria, Bacteroides with three predominant genera: Corynebacterium, Propionibacterium and Staphylococcus (Grice et al. 2008). This microbiota starts to colonize the sterile skin of new-born during the delivery process, the latter being known to influence the skin microbiota composition (Dominguez-Bello et al. 2010; Chu et al. 2017). An immune tolerance against these commensal microorganisms allows a constant colonization of the skin during childhood and is dependent on a combination of individual-related internal and external factors (Capone et al. 2011). At adulthood, the skin microbiota reaches an equilibrium unique for each individual (Schommer and Gallo 2013). Beyond this intra-individual temporal stability, the microbial communities mainly vary across body

This article is protected by copyright. All rights reserved. 
sites (Costello et al. 2009; Oh et al. 2016; Perez Perez et al. 2016). It is now assumed that specific bacteria are associated with moist, dry and sebaceous microenvironments since each site represents an ecological biotope that favors the growth of its own microorganism communities (Grice and Segre 2011; Barnard and Li 2017).

Inter-personal variations of skin microbiota have also been largely reported (Ursell et al. 2012; Rodrigues Hoffmann 2017). Indeed, the composition of human skin microbiota is influenced by multiple factors such as gender, environment, lifestyle and hygiene practices (Fierer et al. 2008; Ying et al. 2015; Prescott et al. 2017; Szabó et al. 2017). Among these factors, the influence of aging on skin microbiota has been studied for many years (Somerville 1969; Leyden et al. 1975). Most published studies about this topic have focused on the physiological changes of skin during aging and how they may affect skin ecology and thus resident microbiota (Wilhelm et al. 1991; Farage et al. 2008). However, the knowledge gathered on skin microbiota was limited to culture-dependent assays, reducing the number of identified species. With the development of next generation sequencing, three recent studies have directly shown that the diversity of skin microbiota was largely affected by skin aging in adult women (Shibagaki et al. 2017; Somboonna et al. 2017) or men (Wilantho et al. 2017). While these studies give new insights in microbial diversity variations with aging, analyses are restricted to Asian skin. To our knowledge, the bacterial diversity between younger and older Caucasian skins has not yet been evaluated.

As skin microbial communities vary among all ethnicities (Leung et al. 2015), results obtained on Asian skin cannot be extrapolated to worldwide populations. It would therefore be of interest to identify potential age-related changes in cutaneous microbiota for each ethnic origin. In this context, the aim of our study was to compare the microbiota diversity between two different age groups of Western European women. For this purpose, bacterial communities, collected from the forehead of each individual, were evaluated based on the 16S rRNA gene sequencing data. Our results show agerelated changes in the diversity of Western European women skin microbiota, thus confirming

This article is protected by copyright. All rights reserved. 
previous data obtained on Asian skin (Shibagaki et al. 2017; Somboonna et al. 2017). This allows us to highlight the bacterial signature on Western European skin across aging.

\section{Material and methods}

\section{Mock communities}

Commercially available microbial mock communities, ZymoBIOMICS ${ }^{\mathrm{m}}$ Microbial Community Standard (lot no. ZRC183430) and ZymoBIOMICS ${ }^{\mathrm{TM}}$ Microbial Community DNA Standard (lot no. ZRC186520) were obtained from ZymoResearch. Microbial Community Standard consists of a mock microbial community in even cell distribution whereas Microbial Community DNA Standard is a mixture of equal volumes of genomic DNA extracted from pure cultures. Microbial standards contain eight bacterial species: $4.6 \%$ Pseudomonas aeruginosa, $10.0 \%$ Escherichia coli, $11.3 \%$ Salmonella enterica, $18.8 \%$ Lactobacillus fermentum, $10.4 \%$ Enterococcus faecalis, $13.3 \%$ Staphyloccocus aureus, 15.9 \% Listeria monocytogenes and $15.7 \%$ Bacillus subtilis. Expected compositions of mock communities were given by the manufacturer.

\section{Skin sample collection}

Samples were obtained from the forehead of 34 Western European women divided into two age groups: 17 younger individuals ranging between 21 and 31 years old (mean $28 \pm 3$ years) and 17 older menopausal individuals ranging between 54 and 69 years old (mean $62 \pm 5$ years). All participants live in Brive-la-Gaillarde (Corrèze, France) or nearby. This study was compliant with the Declaration of Helsinki Principles and it was approved by the institutional review board of SILAB. Subjects were selected carefully to fit in the project's scope. Included subjects were given clear and precise information regarding the outlines of the project. All volunteers signed an informed consent form and declared to be in good health with no current skin infections. They were asked not to receive any antibiotics or antifungals for at least one month prior the study, to keep their hygiene products and skincare habits within the previous 14 days, to remove their makeup and not wash their face and hair or apply any skincare product $12 \mathrm{~h}$ before the sampling.

This article is protected by copyright. All rights reserved. 
Sampling was conducted in a temperature $\left(22 \pm 2^{\circ} \mathrm{C}\right)$ and humidity $(40-60 \%)$ controlled area using a strict procedure of superficial skin swabbing. The middle of the foreheads were gently rubbed on an area of $5 \times 2 \mathrm{~cm}$ using sterile cotton-tipped swabs (FLOQSwabs ${ }^{\mathrm{TM}}$, COPAN) moistened with $0.15 \mathrm{~mol} \mathrm{l}^{-1}$ sodium chloride $(\mathrm{NaCl}), 0.1 \%$ Tween 20 . Cotton tips were immediately broken off into MoBio Power Bead tubes filled with Powerbead ${ }^{\circ}$ solution (Qiagen) and tubes were frozen at $-20^{\circ} \mathrm{C}$ until DNA extraction. Unused swabs and unused buffer tubes without swabs served as negative controls for sampling and DNA isolation.

\section{DNA extraction}

DNA extraction of the Microbial Community Standard and the skin swabs were carried out using the DNeasy PowerLyzer PowerSoil kit (Qiagen) and a Precellys 24 homogenizer (Bertin Technologies) with the following modifications. Efficient cell lysis was ensured by incubating PowerBead tubes at $75^{\circ} \mathrm{C}$ for $15 \mathrm{~min}$ and bead beating at $6,800 \mathrm{rpm}$ for $45 \mathrm{~s}$. Total DNA was eluted in $50 \mu \mathrm{L}$ and stored at $-20^{\circ} \mathrm{C}$ until DNA sequencing. The concentration of extracted DNA was determined using Quant-iT PicoGreen dsDNA Assay Kit (Thermo-Fisher scientific) according to manufacturer's instructions.

\section{$16 S$ library preparation and sequencing}

Hypervariable regions V3-V4 of the bacterial 16S rRNA were amplified during a first targeted PCR using primers containing Illumina adapters as described in Illumina's 16S Metagenomics Protocol (Part \#15044223 Rev. B): primers forward TCGTCGGCAGCGTCAGATGTGTAT AAGAGACAGCCTACGGGNGGCWGCAG and primers reverse GTCTCGTGGGCTCGG AGATGTGTATAAGAGACAGGACTACHVGGGTATCTAATCC (the adapters are highlighted in bold). $10 \mu \mathrm{L}$ of total DNA extract were used for a $50 \mu$ I PCR reaction containing 1X KAPA HiFi HotStart ReadyMix (KAPA Biosystem), $0.8 \mu \mathrm{moll}^{-1}$ of each primer and PCR grade water (Sigma-Aldrich). PCR was performed on a Mastercycler EP Gradient thermocycler (Eppendorf) by using the following conditions: an initial denaturation step at $95^{\circ} \mathrm{C}$ for $3 \mathrm{~min}$ followed by 30 cycles of denaturation at $95^{\circ} \mathrm{C}$ for $30 \mathrm{~s}$, annealing at $58^{\circ} \mathrm{C}$ for $30 \mathrm{~s}$, and extension at $72^{\circ} \mathrm{C}$ for $30 \mathrm{~s}$, and a final extension step at

This article is protected by copyright. All rights reserved. 
$72^{\circ} \mathrm{C}$ for 5 min. A second indexing PCR was performed to uniquely barcode each sample with $5^{\prime}$ and 3' Nextera XT dual-indices (Illumina). Specifically, PCR reaction contained 1X KAPA HiFi HotStart ReadyMix (KAPA Biosystem), $5 \mu$ of each primer, PCR grade water (Sigma-Aldrich) and $5 \mu \mathrm{L}$ of the targeted PCR product used as the template in a $50 \mu \mathrm{l}$ volume PCR reaction. Cycling conditions were the same as the targeted PCR, except for the annealing temperature reduced at $55^{\circ} \mathrm{C}$, with only eight amplification cycles. After each PCR, products were purified using Agencourt AMPure XP magnetic beads (Beckman Coulter) according to manufacturer's protocol and DNA amplification was checked on a $2 \%$ agarose gel. Final DNA concentration was measured using Quant-iT PicoGreen dsDNA Assay Kit (Thermo-Fisher scientific) and was adjusted to $4 \mathrm{nmol} \mathrm{I}^{-1}, 5 \mu \mathrm{L}$ of each sample were then pooled together. Pooled samples were denatured with $0.2 \mathrm{moll}^{-1}$ of $\mathrm{NaOH}$, further diluted to $6 \mathrm{pmol} \mathrm{I}^{-1}$ and combined with $15 \%(\mathrm{v} / \mathrm{v})$ of a denatured PhiX solution at $6 \mathrm{pmol}^{-1}$. Samples were paired end sequenced $(2 \times 251)$ on the Illumina MiSeq sequencing platform using a MiSeq Reagent Kit v3 (600 cycles) (Illumina) according to a standard protocol. After sequencing, sequences were demultiplexed, and the generated FASTQ were used for downstream data analysis.

\section{Bioinformatics analysis}

Sequencing dataset were processed with FROGS pipeline supported by the Galaxy web-based platform (Escudié et al. 2017). Forward and reverse reads of paired-end raw sequences were trimmed against primer sequences using CUTADAPT (v1.8.3), merged using FLASH (v1.2.11) and dereplicated. During this step, quality control was applied by discarding reads shorter than $400 \mathrm{bp}$ or displaying a rate of mismatch in the overlap region $>10 \%$. Similar high quality sequences were de novo clustered into operational taxonomic units (OTUs) using SWARM clustering method (v2.1.1) (Mahé et al. 2014) with an aggregation distance of 3. Chimeric OTUs were identified by the UCHIME algorithm (v1.1.3) and removed. Only OTUs that made up to $0.005 \%$ or more of the total sequences were considered (Bokulich et al. 2013). A representative sequence for each OTU was used to assign taxonomy using BLASTN+ (v2.2.30) against a curated version of the SILVA 16S rRNA database (release 128 with sequences displaying a Pintail value of 100) with a minimum identity and coverage

This article is protected by copyright. All rights reserved. 
threshold of $96 \%$ and $99 \%$ respectively. Annotated OTUs were used to determine the relative abundance of bacterial phyla and genera in each sample. Representative sequences from each OTU were aligned by using PyNAST and a phylogenetic tree was inferred by using FastTree (v2.1.10). Rarefaction curves and analyses of alpha and beta diversity were performed in R language (v3.4.2) using the phyloseq and ggplot2 package in Rstudio (v1.1.383). To avoid biases generated by differences in sequencing depth, the OTU table was rarified to an even depth of 35,000 sequences for diversity analysis. Alpha diversity was evaluated by calculating three indices including observed OTU numbers, Chao1-estimated OTU numbers and Shannon index. Beta diversity was assessed by calculating UniFrac distance among the samples (weighted and unweighted) from OTU table. Principal coordinates analysis (PCOA) of beta diversity was used for cluster analysis between age groups. Good's coverage was calculated using Qiime software (v1.9.1). A linear discriminant analysis (LDA) with effect size (LEfSe) (Segata et al. 2011) generated in Galaxy was used to identify differentially contribution of genera between groups. A $P$ value $<0.05$ was considered as statistical significance and the threshold of LDA score was set to 3.0.

\section{Statistical analysis}

Statistical significance was determined using the two sample t-test or the Wilkoxon's rank sum test, according to data analysed. Analysis was performed using Statsgraphics centurion 18 software.

Permutational multivariate analysis of variance (PERMANOVA) was applied to analyse the differences in microbiota structure between age groups using the "adonis" function in the Vegan package (Rstudio). $\mathrm{R}^{2}$ indicates the percentage of variance explained by the age parameter. Significance was reported when $P<0.05$.

This article is protected by copyright. All rights reserved. 


\section{Results}

\section{Validation of analysis pipeline}

We first applied our library preparation and bioinformatics analysis methods to two commercially available microbial community standards (Fig. 1). A very high Pearson correlation coefficient was observed between the Mock Community and Mock DNA $(r=0.99)$, revealing no bias associated with the nucleic acid extraction method employed. Furthermore, every genus present in microbial community standards was identified with a strong correlation in the abundance level between the expected and the observed composition for the Mock Community $(r=0.87)$ and Mock DNA ( $r=$ $0.89)$, thus validating our analysis pipeline.

\section{Overview of sequencing data}

A total of $2,379,018$ reads with an average length of $417 \mathrm{bp}$ were obtained from the 34 skin samples after filtering the data. Mean sequence reads per sample was of $64,531 \pm 16,919$ and $69,971 \pm 16,909$ for younger and older age group respectively, with a minimum of 35,608 reads (for details in sequence distribution see Table S1). To avoid bias due to sequencing depth, analysis were conducted on 35,000 reads for each sample. These reads were classified in 310 OTUs obtained among all samples (all of them were classified as bacteria), revealing the presence of seven phyla and 77 genera.

\section{Age-related differences in alpha diversity}

Alpha diversity describes the diversity within a sample. Rarefaction curves of the observed OTU numbers as a function of the sequence reads were calculated. All of them tended to reach a plateau, indicating the number of OTUs we analysed for each sample was sufficient to exhibit the full species diversity (Fig. 2a). All samples also displayed a Good's coverage value > 0.99 (Table S1), confirming a strong sequencing depth. Alpha diversity between the two age groups was analysed using both observed and chao1-estimated OTU numbers - i.e. two metrics reflecting species richness - and the Shannon index - i.e. an ecological measure of community richness and evenness.

This article is protected by copyright. All rights reserved. 
No significant difference was found in the number of observed or chao1-estimated OTU revealing no difference in the richness within samples of the two age groups (Fig. 2b,c). Conversely, Shannon alpha diversity index significantly increased in older age group $(1.74 \pm 0.35)$ compared to younger age group (1.30 \pm 0.44 ) (Fig. $2 \mathrm{~d}$ ), thus indicating an age-related modification in the distribution of bacterial communities. Interestingly, Shannon index appears close to 1, reflecting a low diversity profile of the forehead skin.

\section{Age-related differences in beta diversity}

Beta diversity considers the overall structural difference and similarity between different microbial communities. Beta diversity among samples was assessed by calculating both weighted (considers relative abundance of OTUs) or unweighted (presence/absence of OTUs) phylogenetic UniFrac distances at the OTU level (Lozupone et al. 2007). PCoA of weighted UniFrac distance revealed a distinct clustering in samples of both age groups (Fig. $3 a ; r^{2}=0.11602, P=0.0082$ ) while no clustering was found for unweighted UniFrac distances (Fig. 3b; $\mathrm{r}^{2}=0.04983, \mathrm{P}=0.0784$ ). These data suggest that age-related modifications in the beta diversity may be primarily due to a variation in the most abundant OTUs rather than to differences in the type of bacterial lineages present. Moreover, greater dispersion of samples from the older age group based on weighted UniFrac distances revealed a higher dissimilarity within older individuals.

\section{Taxonomic changes in skin microbiota between the two age groups}

Taxonomic changes in the age-related diversification were next investigated at both phylum and genus levels. The bacterial community of forehead skin was dominated by four phyla: Actinobacteria, Firmicutes, Proteobacteria and Bacteroidetes representing more than $99 \%$ of the total sequences across the two age groups. (Fig. 4a). The proportion of Actinobacteria in older individuals (mean: $46.7 \%$ ) was significantly lower than in younger ones (59.2\%). In contrast, the phylum Proteobacteria was significantly more abundant in older age group (13.9\%) than in younger age group (3.5\%) (Figure S1).

This article is protected by copyright. All rights reserved. 
Seventy-seven genera were detected in the dataset, and 10 of them were found in larger proportion with an average abundance $>0.5 \%$ (Fig. 4b). Among them, Propionibacterium and Staphyloccocus genera represent more than $80 \%$ of the total reads. Propionibacterium were significantly less abundant in the older age group (40.6\%) compared to the younger age group (56.6\%), whereas a markedly higher proportion of Corynebacterium was found in older age group (5.0\% compared to $0.8 \%$ in the younger age group). In addition, a non-significant trend toward an increase in Enhydrobacter and Acinetobacter was also observed in older individuals, contributing to the higher abundance of Proteobacteria in this age group. Moreover, the composition of the "others" category (unknown genera or genera with $<0.5 \%$ mean relative abundance) was significantly higher in the older (14.2\%) than in the younger age group (5.1\%) (Figure S2).

To go even further, LEfSe tool was used to measure the contribution of each taxon to the previously identified differences between the two age groups (Fig. 4c,d). As expected, a high contribution of the genera Propionibacterium (higher in younger age group: LDA score 4.9) and Corynebacterium (higher in older age group: LDA score 4.3) was found. Additionally, five other genera were identified (LDA scores $>3.0$ ), contributing to the differences observed in "others" group. Two of them, an unknown genus in the order Neisseriaceae (LDA score 4.6) and Halomonas (LDA score 4.2), both belonging to the Proteobacteria, contribute to the age-related difference observed for this phylum.

This article is protected by copyright. All rights reserved. 


\section{Discussion}

In the present study, we demonstrated that the diversity and taxonomic composition of skin microbiota of Western European women are strongly influenced during chronological aging. This study compared the skin microbiota of Western European individuals belonging to two age groups, albeit we note that the selected cohort constitutes only a part of this population. As it was shown that different environmental exposure or diet could influence microbiota in the gut (Zhang et al. 2015), this skin-based study should be extended to other Caucasian groups to take into account different lifestyles which may coexist within this population. Moreover, by focusing on women, this study analysed age-related microbiota variations, regardless of gender influence (Ying et al. 2015). Nevertheless, it would also be interesting to perform the same study on male volunteers. The analysed body site - i.e. forehead - was selected according to two main criteria. Firstly, although aging affects all organs, one's age is generally assessed by different features observed on the face, which plays an important role in human identity and social interactions (Nkengne et al. 2008; Samson et al. 2010). Secondly, a recent study conducted by Shibagaki and collaborators (Shibagaki et al. 2017), demonstrated a high similarity on microbiota diversity between different face sites - i.e. cheeks or forehead.

Data revealed higher alpha diversity on the skin of older individuals compared to younger individuals with a significant age-related change in the distribution but not in the richness of the forehead skin microbiota. Nevertheless, the richness of the samples tends to be higher in older subjects compared to the younger ones. This result is slightly different of previous studies conducted on Thai and Japanese women reporting a strong skin microbiome richness on older individuals (Shibagaki et al. 2017; Wilantho et al. 2017). This discrepancy could be explained by the regions of the 16S rDNA selected - i.e. V3-V4 in this study - (Meisel et al. 2016), but also by the different environment in which each panel of volunteers evolves (Leung et al. 2015). In parallel, results obtained for the Shannon index reflect a low diversity profile of the forehead skin, partly explained

This article is protected by copyright. All rights reserved. 
by an overrepresentation of Propionibacterium and Staphylococcus. This is consistent with previous studies showing lower bacterial diversity in sebaceous areas such as forehead, cheek or scalp than dry areas such as forearm (Grice et al. 2009; Oh et al. 2016; Perez Perez et al. 2016). Additionally, beta diversity examination revealed that young and aged skins share the same species but in different relative abundances. Altogether, these results suggest a higher proportion of minor OTUs on older compared to younger skin.

These results mirrored those obtained by taxonomic classification, showing both an increase of Proteobacteria and a decrease of Actinobacteria on aged skin. Similar results were reported on palms, forearm, forehead, cheek and scalp of male and female Asian volunteers (Shibagaki et al. 2017; Somboonna et al. 2017; Wilantho et al. 2017). Interestingly, a study suggests that Proteobacteria are temporary environmental contaminants of the human skin (Cosseau et al. 2016). Moreover, this phylum seems to be more abundant in psoriatic lesions and chronic wounds than in healthy skin (Han et al. 2011; Fahlén et al. 2012). Like Actinobacteria, the older age group showed a significant decrease of Propionibacterium. Several studies reported that Propionibacterium is a lipophilic resident of sebum-rich regions (Costello et al. 2009; Grice et al. 2009; Bouslimani et al. 2015). Consistent with these results, changes in hormonal levels already described during menopausal state and leading to lower sebum production may explain the reduction of Propionibacterium spp. observed in older age group (Bensaleh et al. 2006). Since Propionibacterium spp. was recently described as producing an enzyme with antioxidant capacities (Allhorn et al. 2016), its lower abundance in the older age group might partly contribute to the increase of oxidative stress that is characteristic of aged skin (Lephart 2016). Within Actinobacteria we also observed a striking increase of Corynebacterium spp. in older age group, as previously reported on axillary fossae (Li et al. 2014). While commensal, this genus could become pathogen as several studies have reported the link between Corynebacterium spp. increase and cutaneous infections, in conjunction with skin barrier defects (Cogen et al. 2008; van Rensburg et al. 2015). As some species share the same V3-V4 regions, our methodology does not allow an analysis at species level for all the genera. To go even

This article is protected by copyright. All rights reserved. 
further in the characterization of skin from aged individuals and its microbiota, it would be of interest to sequence other hypervariable regions or to perform whole genome shotgun sequencing.

While our study does not elucidate the reason of microbiota shift with age, it seems that modifications in skin physiology during the process of aging, such as sebum secretion, hydration, $\mathrm{pH}$ level and lipid composition, could predict microbiota changes (Mukherjee et al. 2016). Moreover, innate immune defenses of the skin are weakened with age, thus increasing susceptibility to pathogenic invasions and further impairment of barrier function (Vukmanovic-Stejic et al. 2011). Hence, skin of young individuals may have a very important role in selecting commensal bacteria allowing overrepresentation of Firmicutes and Actinobacteria and thus limiting the bacterial diversity. In contrast, skin of aged individuals, due to physiological and immune changes, may relieve this selective pressure, so that rare opportunistic bacteria such as Proteobacteria may colonise the skin, leading to a higher diversity. If we hypothesise that the shift in the skin microbiota is a consequence rather than a cause of the aging process, it may explain the greater inter-personal variation observed within individuals of the older group related to the wide range of aging kinetics of each individual.

In conclusion, this study presented age-related changes in the skin microbiota diversity of Western European women. It is suggested that this imbalance in the skin microbiota is linked to physiological changes in skin that occur during chronological aging or following external factors. Hence, the state of skin microbiota could become a readout predicting the progression of aging on skin. While deepen our knowledge on skin modifications during aging, this study will help to develop new approaches in order to rebalance skin microbiota and prevent age-related skin disorders.

This article is protected by copyright. All rights reserved. 


\section{Conflict of interest}

The authors have no conflict of interest to declare.

\section{Bibliography}

Allhorn, M., Arve, S., Brüggemann, H. and Lood, R. (2016). A novel enzyme with antioxidant capacity produced by the ubiquitous skin colonizer Propionibacterium acnes. Sci. Rep. 6:36412.

Baldwin, H.E., Bhatia, N.D., Friedman, A., Eng, R.M. and Seite, S. (2017). The Role of Cutaneous Microbiota Harmony in Maintaining a Functional Skin Barrier. J. Drugs Dermatol. JDD 16:12-18.

Barnard, E. and Li, H. (2017). Shaping of cutaneous function by encounters with commensals. J. Physiol. 595:437-450.

Bensaleh, H., Belgnaoui, F.Z., Douira, L., Berbiche, L., Senouci, K. and Hassam, B. (2006). [Skin and menopause]. Ann. Endocrinol. 67:575-580.

Bokulich, N.A., Subramanian, S., Faith, J.J., Gevers, D., Gordon, J.I., Knight, R., Mills, D.A., et al. (2013). Quality-filtering vastly improves diversity estimates from Illumina amplicon sequencing. Nat. Methods 10:57-59.

Bouslimani, A., Porto, C., Rath, C.M., Wang, M., Guo, Y., Gonzalez, A., Berg-Lyon, D., et al. (2015). Molecular cartography of the human skin surface in 3D. Proc. Natl. Acad. Sci. U. S. A. 112:E21202129.

Byrd, A.L., Belkaid, Y. and Segre, J.A. (2018). The human skin microbiome. Nat. Rev. Microbiol. 16:143-155.

Capone, K.A., Dowd, S.E., Stamatas, G.N. and Nikolovski, J. (2011). Diversity of the human skin microbiome early in life. J. Invest. Dermatol. 131:2026-2032.

Chu, D.M., Ma, J., Prince, A.L., Antony, K.M., Seferovic, M.D. and Aagaard, K.M. (2017). Maturation of the infant microbiome community structure and function across multiple body sites and in relation to mode of delivery. Nat. Med. 23:314-326.

Cogen, A.L., Nizet, V. and Gallo, R.L. (2008). Skin microbiota: a source of disease or defence? Br. J. Dermatol. 158:442-455.

Cosseau, C., Romano-Bertrand, S., Duplan, H., Lucas, O., Ingrassia, I., Pigasse, C., Roques, C., et al. (2016). Proteobacteria from the human skin microbiota: Species-level diversity and hypotheses. One Health 2:33-41.

Costello, E.K., Lauber, C.L., Hamady, M., Fierer, N., Gordon, J.I. and Knight, R. (2009). Bacterial Community Variation in Human Body Habitats Across Space and Time. Science 326:1694-1697.

Dominguez-Bello, M.G., Costello, E.K., Contreras, M., Magris, M., Hidalgo, G., Fierer, N. and Knight, R. (2010). Delivery mode shapes the acquisition and structure of the initial microbiota across multiple body habitats in newborns. Proc. Natl. Acad. Sci. U. S. A. 107:11971-11975.

This article is protected by copyright. All rights reserved. 
Dréno, B., Araviiskaia, E., Berardesca, E., Gontijo, G., Sanchez Viera, M., Xiang, L.F., Martin, R., et al. (2016). Microbiome in healthy skin, update for dermatologists. J. Eur. Acad. Dermatol. Venereol. JEADV 30:2038-2047.

Escudié, F., Auer, L., Bernard, M., Mariadassou, M., Cauquil, L., Vidal, K., Maman, S., et al. (2017). FROGS: Find, Rapidly, OTUs with Galaxy Solution. Bioinforma. Oxf. Engl.

Fahlén, A., Engstrand, L., Baker, B.S., Powles, A. and Fry, L. (2012). Comparison of bacterial microbiota in skin biopsies from normal and psoriatic skin. Arch. Dermatol. Res. 304:15-22.

Farage, M.A., Miller, K.W., Elsner, P. and Maibach, H.I. (2008). Functional and physiological characteristics of the aging skin. Aging Clin. Exp. Res. 20:195-200.

Fierer, N., Hamady, M., Lauber, C.L. and Knight, R. (2008). The influence of sex, handedness, and washing on the diversity of hand surface bacteria. Proc. Natl. Acad. Sci. U. S. A. 105:17994-17999.

Fredricks, D.N. (2001). Microbial ecology of human skin in health and disease. J. Investig. Dermatol. Symp. Proc. 6:167-169.

Ganju, P., Nagpal, S., Mohammed, M.H., Nishal Kumar, P., Pandey, R., Natarajan, V.T., Mande, S.S., et al. (2016). Microbial community profiling shows dysbiosis in the lesional skin of Vitiligo subjects. Sci. Rep. 6:18761.

Grice, E.A., Kong, H.H., Conlan, S., Deming, C.B., Davis, J., Young, A.C., NISC Comparative Sequencing Program, et al. (2009). Topographical and temporal diversity of the human skin microbiome. Science 324:1190-1192.

Grice, E.A., Kong, H.H., Renaud, G., Young, A.C., NISC Comparative Sequencing Program, Bouffard, G.G., Blakesley, R.W., et al. (2008). A diversity profile of the human skin microbiota. Genome Res. 18:1043-1050.

Grice, E.A. and Segre, J.A. (2011). The skin microbiome. Nat. Rev. Microbiol. 9:244-253.

Han, A., Zenilman, J.M., Melendez, J.H., Shirtliff, M.E., Agostinho, A., James, G., Stewart, P.S., et al. (2011). The importance of a multifaceted approach to characterizing the microbial flora of chronic wounds. Wound Repair Regen. 19:532-541.

Kong, H.H., Oh, J., Deming, C., Conlan, S., Grice, E.A., Beatson, M.A., Nomicos, E., et al. (2012). Temporal shifts in the skin microbiome associated with disease flares and treatment in children with atopic dermatitis. Genome Res. 22:850-859.

Lephart, E.D. (2016). Skin aging and oxidative stress: Equol's anti-aging effects via biochemical and molecular mechanisms. Ageing Res. Rev. 31:36-54.

Leung, M.H.Y., Wilkins, D. and Lee, P.K.H. (2015). Insights into the pan-microbiome: skin microbial communities of Chinese individuals differ from other racial groups. Sci. Rep. 5:11845.

Leyden, J.J., McGinley, K.J., Mills, O.H. and Kligman, A.M. (1975). Age-related changes in the resident bacterial flora of the human face. J. Invest. Dermatol. 65:379-381.

Li, W., Han, L., Yu, P., Ma, C., Wu, X. and Xu, J. (2014). Nested PCR-denaturing gradient gel electrophoresis analysis of human skin microbial diversity with age. Microbiol. Res. 169:686-692.

This article is protected by copyright. All rights reserved. 
Lozupone, C.A., Hamady, M., Kelley, S.T. and Knight, R. (2007). Quantitative and qualitative beta diversity measures lead to different insights into factors that structure microbial communities. Appl. Environ. Microbiol. 73:1576-1585.

Madison, K.C. (2003). Barrier function of the skin: 'la raison d'être' of the epidermis. J. Invest. Dermatol. 121:231-241.

Mahé, F., Rognes, T., Quince, C., de Vargas, C. and Dunthorn, M. (2014). Swarm: robust and fast clustering method for amplicon-based studies. PeerJ 2:e593.

Meisel, J.S., Hannigan, G.D., Tyldsley, A.S., SanMiguel, A.J., Hodkinson, B.P., Zheng, Q. and Grice, E.A. (2016). Skin Microbiome Surveys Are Strongly Influenced by Experimental Design. J. Invest.

Dermatol. 136:947-956.

Mukherjee, S., Mitra, R., Maitra, A., Gupta, S., Kumaran, S., Chakrabortty, A. and Majumder, P.P. (2016). Sebum and Hydration Levels in Specific Regions of Human Face Significantly Predict the Nature and Diversity of Facial Skin Microbiome. Sci. Rep. 6:36062.

Nkengne, A., Bertin, C., Stamatas, G.N., Giron, A., Rossi, A., Issachar, N. and Fertil, B. (2008). Influence of facial skin attributes on the perceived age of Caucasian women. J. Eur. Acad. Dermatol. Venereol. JEADV 22:982-991.

Oh, J., Byrd, A.L., Park, M., NISC Comparative Sequencing Program, Kong, H.H. and Segre, J.A. (2016). Temporal Stability of the Human Skin Microbiome. Cell 165:854-866.

Perez Perez, G.I., Gao, Z., Jourdain, R., Ramirez, J., Gany, F., Clavaud, C., Demaude, J., et al. (2016). Body Site Is a More Determinant Factor than Human Population Diversity in the Healthy Skin Microbiome. PloS One 11:e0151990.

Prescott, S.L., Larcombe, D.-L., Logan, A.C., West, C., Burks, W., Caraballo, L., Levin, M., et al. (2017). The skin microbiome: impact of modern environments on skin ecology, barrier integrity, and systemic immune programming. World Allergy Organ. J. 10:29.

van Rensburg, J.J., Lin, H., Gao, X., Toh, E., Fortney, K.R., Ellinger, S., Zwickl, B., et al. (2015). The Human Skin Microbiome Associates with the Outcome of and Is Influenced by Bacterial Infection. mBio 6:e01315-1315.

Rocha, M.A. and Bagatin, E. (2017). Skin barrier and microbiome in acne. Arch. Dermatol. Res.

Rodrigues Hoffmann, A. (2017). The cutaneous ecosystem: the roles of the skin microbiome in health and its association with inflammatory skin conditions in humans and animals. Vet. Dermatol. 28:60e15.

Samson, N., Fink, B. and Matts, P.J. (2010). Visible skin condition and perception of human facial appearance. Int. J. Cosmet. Sci. 32:167-184.

Sanford, J.A. and Gallo, R.L. (2013). Functions of the skin microbiota in health and disease. Semin. Immunol. 25:370-377.

Schommer, N.N. and Gallo, R.L. (2013). Structure and function of the human skin microbiome. Trends Microbiol. 21:660-668.

This article is protected by copyright. All rights reserved. 
Segata, N., Izard, J., Waldron, L., Gevers, D., Miropolsky, L., Garrett, W.S. and Huttenhower, C. (2011). Metagenomic biomarker discovery and explanation. Genome Biol. 12:R60.

Shibagaki, N., Suda, W., Clavaud, C., Bastien, P., Takayasu, L., lioka, E., Kurokawa, R., et al. (2017). Aging-related changes in the diversity of women's skin microbiomes associated with oral bacteria. Sci. Rep. 7:10567.

Somboonna, N., Wilantho, A., Srisuttiyakorn, C., Assawamakin, A. and Tongsima, S. (2017). Bacterial communities on facial skin of teenage and elderly Thai females. Arch. Microbiol. 199:1035-1042.

Somerville, D.A. (1969). The normal flora of the skin in different age groups. Br. J. Dermatol. 81:248258.

Szabó, K., Erdei, L., Bolla, B.S., Tax, G., Bíró, T. and Kemény, L. (2017). Factors shaping the composition of the cutaneous microbiota. Br. J. Dermatol. 176:344-351.

Tett, A., Pasolli, E., Farina, S., Truong, D.T., Asnicar, F., Zolfo, M., Beghini, F., et al. (2017). Unexplored diversity and strain-level structure of the skin microbiome associated with psoriasis. NPJ Biofilms Microbiomes 3:14.

Ursell, L.K., Clemente, J.C., Rideout, J.R., Gevers, D., Caporaso, J.G. and Knight, R. (2012). The interpersonal and intrapersonal diversity of human-associated microbiota in key body sites. J. Allergy Clin. Immunol. 129:1204-1208.

Vukmanovic-Stejic, M., Rustin, M.H.A., Nikolich-Zugich, J. and Akbar, A.N. (2011). Immune responses in the skin in old age. Curr. Opin. Immunol. 23:525-531.

Wilantho, A., Deekaew, P., Srisuttiyakorn, C., Tongsima, S. and Somboonna, N. (2017). Diversity of bacterial communities on the facial skin of different age-group Thai males. PeerJ 5:e4084.

Wilhelm, K.P., Cua, A.B. and Maibach, H.I. (1991). Skin aging. Effect on transepidermal water loss, stratum corneum hydration, skin surface pH, and casual sebum content. Arch. Dermatol. 127:18061809.

Ying, S., Zeng, D.-N., Chi, L., Tan, Y., Galzote, C., Cardona, C., Lax, S., et al. (2015). The Influence of Age and Gender on Skin-Associated Microbial Communities in Urban and Rural Human Populations. PloS One 10:e0141842.

Zhang, J., Guo, Z., Xue, Z., Sun, Z., Zhang, M., Wang, L., Wang, G., et al. (2015). A phylo-functional core of gut microbiota in healthy young Chinese cohorts across lifestyles, geography and ethnicities. ISME J. 9:1979-1990.

This article is protected by copyright. All rights reserved. 


\section{Acknowledgements}

We thank all the volunteers for skin samples collection.

\section{Availability of data}

The sequence data set was deposited to the NCBI/EBI/DDBJ/Sequence Read Archive (submission no. SUB3375546; http://www.ncbi.nlm.nih.gov/bioproject/427175).

This article is protected by copyright. All rights reserved. 


\section{Figures legends}

Figure 1: Taxonomic composition of Mock community standards using the MiSeq sequencing pipeline. Stacked bar charts showing the relative abundance at the genus level within the Mock DNA and the Mock community standard obtained using the Miseq sequencing pipeline, compared to the expected composition for the mock communities. DNA from Mock community standard was extracted and analysed thereafter. The Pearson correlation coefficients between the expected relative abundance and the Mock DNA and Mock community composition or between both Mock community standards are indicated on top of the bar graph. ( $\square$ ) Bacillus; ( $\quad$ ) Enterococcus; ( $\square$ ) Escherichia; ( ) Lactobacillus; ( ) Listeria; ( ) Pseudomonas; ( $\quad$ ) Salmonella; ( $)$ Staphylococcus; ( ) Others genera.

Figure 2: Comparison of forehead bacterial alpha diversity between younger and older age group. (a) Rarefaction curves calculated on samples from younger (blue) and older (red) subjects. Box and whiskers plots showing observed (b) as well as chao1-estimated (c) richness and Shannon diversity index (d) for each group, calculated at the OTU level. Dots indicate the outliers. $* * P<0.01$ using Wilkoxon's rank sum test.

Figure 3: Comparison of the bacterial community beta diversity between younger and older age group. Principal coordinates analysis (PCoA) of bacterial communities from forehead samples at the OTU level using weighted (wUniFrac) (a) and unweighted (UniFrac) (b) UniFrac distances according to younger (blue) and older (red) age groups. The percentage of the variation attributed to an axis is indicated.

Figure 4: Taxonomic analysis of forehead microbiota in younger and older subjects group. Stacked bar charts showing the relative abundance of the four most prevalent bacterial phyla (a), ( $\square$ )

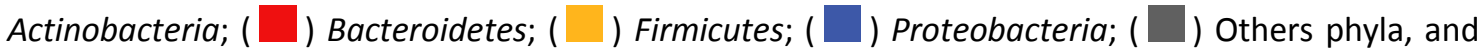
the 10 most prevalent genera (b), ( $($ ) Corynebacterium; ( $)$ Lawsonella; (

This article is protected by copyright. All rights reserved. 


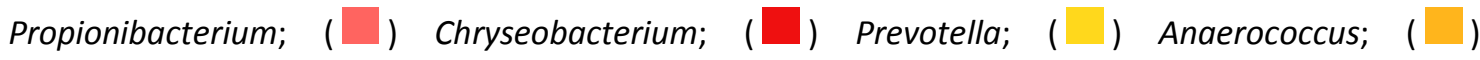
Staphylococcus; ( $\square$ ) Streptococcus; ( $\square$ ) Acinetobacter; ( $\square$ ) Enhydrobacter; ( $\square$ ) Others genera, in the forehead microbiota samples. Other phyla and unknown genera or genera with $<0.5 \%$ mean relative abundance are grouped into the category "others". Significative difference between taxa are shown in Figures S1, S2. (c) Linear discriminant analysis effect size (LEfSe) between older (red) and younger (green) age group $(P<0.05$; LDA score $>3.0)$. (d) Cladogram plotted from LEfSe analysis. Nodes represent phylogenetic levels from domain to genus (from inside out). Each node diameter is proportional to the taxon's abundance and is colored by age groups (red for older and green for younger age group) for which it is significantly more abundant. a: Corynebacterium; b: Arthrobacter; c: Propionibacterium; d: Propionibacteriaceae; e: Propionibacteriales; f: Actinobacteria; g: Sneathia; h: unknown genus; i: Neisseriaceae; j: Neisseriales; k: Betaproteobacteria; I: Halomonas; m: Halomonadaceae; n: Oceanospirillales; o: Moraxellaceae; p: Pseudomonadales.

This article is protected by copyright. All rights reserved. 
Legends for the Supporting Information

Table S1: Metadata, sequencing and alpha diversity statistics of 34 Western European women.

Figure S1: Comparison of the taxonomic composition of the forehead microbiota between the older and younger age group at the phylum level. Box and whiskers plots showing the relative abundance of the four major phyla on the forehead microbiota of the older and younger age group. * $P<0.05 * * P<0.01$ using student t-test or Wilkoxon's rank sum test chosen according to data analysed.

Figure S2: Comparison of the taxonomic composition of the forehead microbiota between the older and younger age group at the genus level. Box and whiskers plots showing the relative abundance of the 10 major genera on the forehead microbiota of the older and younger age group. ** $P<0.05 * * * P<0.01$ using student t-test or Wilkoxon's rank sum test chosen according to data analysed.

This article is protected by copyright. All rights reserved. 

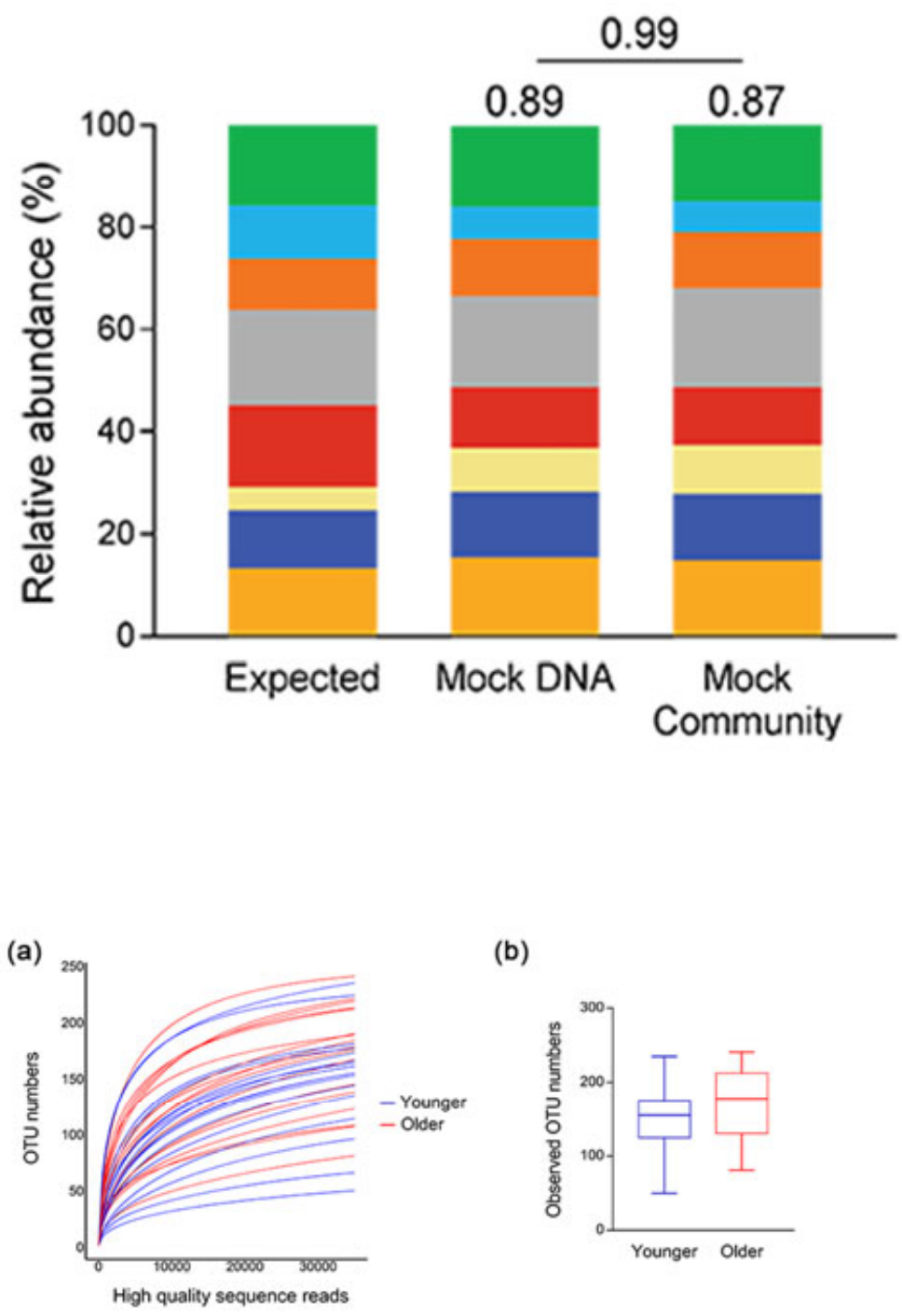

(b)

(c)

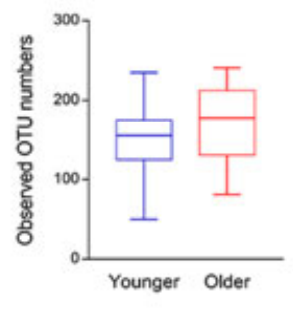

(d)
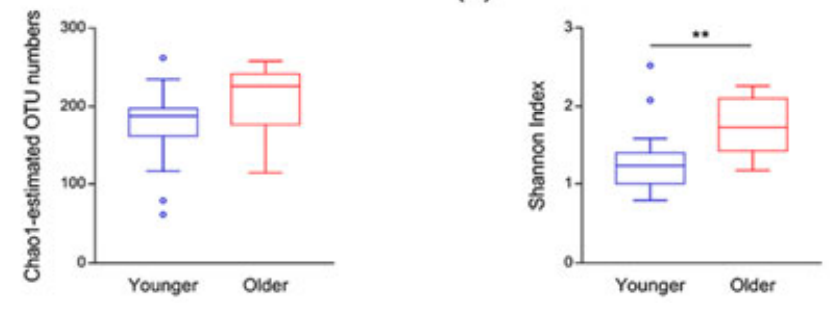

This article is protected by copyright. All rights reserved. 


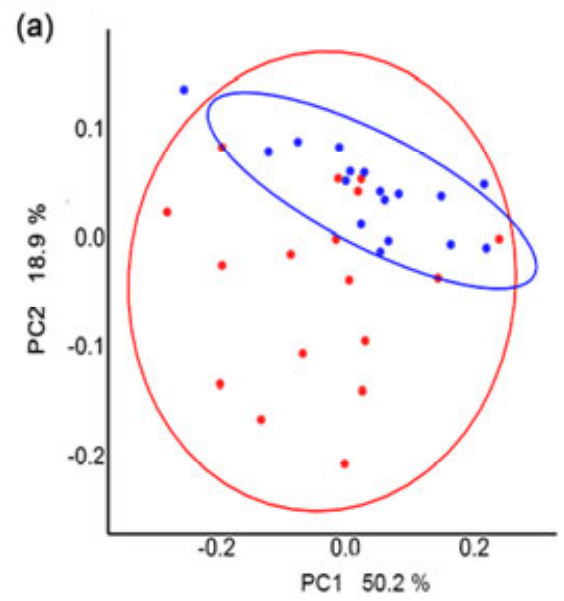

(a)

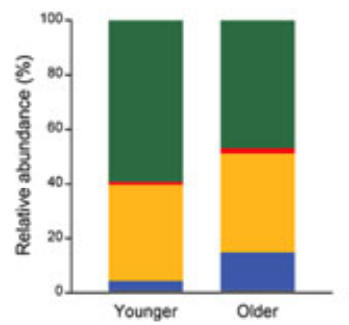

(c)

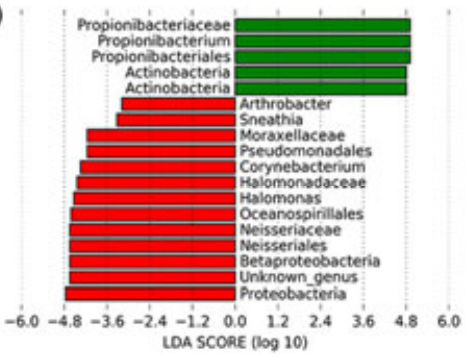

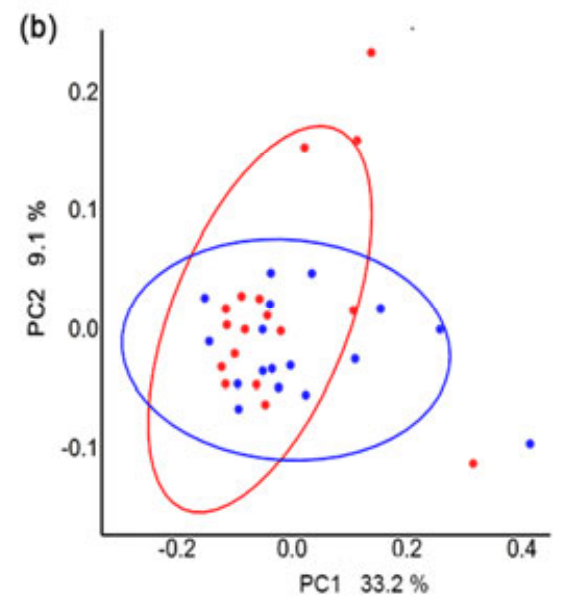

(b)

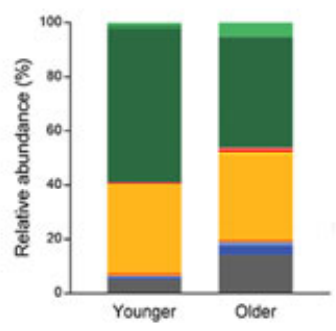

(d)

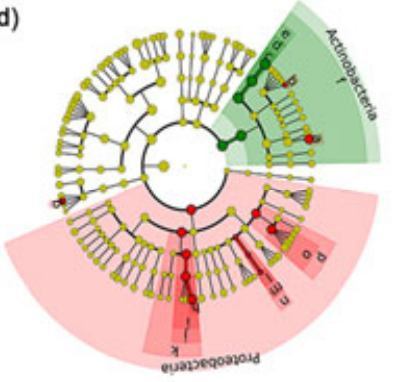

This article is protected by copyright. All rights reserved.

Jugé, R., Rouaud-Tinguely, Breugnot, Servaes, Grimaldi, Roth, M.-P., Coppin, H., Closs (2018). 